\title{
Finite-Time Boundedness Control of Time-Varying Descriptor Systems
}

\author{
Xiaoming Su, ${ }^{1}$ Yali Zhi, ${ }^{1}$ and Qingling Zhang ${ }^{2}$ \\ ${ }^{1}$ School of Science, Shenyang University of Technology, Shenyang 110870, China \\ ${ }^{2}$ School of Science, Northeastern University, Shenyang 110004, China \\ Correspondence should be addressed to Xiaoming Su; suxm@sut.edu.cn
}

Received 29 August 2013; Accepted 2 December 2013

Academic Editor: Yijing Wang

Copyright (C) 2013 Xiaoming Su et al. This is an open access article distributed under the Creative Commons Attribution License, which permits unrestricted use, distribution, and reproduction in any medium, provided the original work is properly cited.

\begin{abstract}
This paper mainly studies a control problem of finite-time boundedness of time-varying descriptor systems. Firstly, a sufficient and necessary condition of finite-time stability is given, then a sufficient condition of finite-time boundedness for time-varying descriptor systems is given. Secondly, we analyze the finite-time boundedness control problem and design the finite-time state feedback controller; the controller is given based on LMIs for time-varying descriptor systems and time-varying uncertain descriptor systems, respectively. Finally, a numerical example is given to prove the effectiveness of the method.
\end{abstract}

\section{Introduction}

Recently, descriptor systems (which are also known as singular systems, semistate systems, systems of differential-algebraic equations, or generalized state space systems.) theory has been well studied since they are very important from the engineering point of view. Let the time-varying descriptor systems be described as $E(t) \dot{x}(t)=A(t) x(t)+B(t) u(t)+G(t) \omega(t)$. They arise naturally in many physical applications such as electrical networks, aircraft and robot dynamics, neutral delay and large-scale systems, economics and optimization problem, biology, constrained mechanics, as result of partial discretization of partial differential equation, and so forth. The timevarying descriptor system has the common properties of general descriptor system and some special properties. In control theory, the time-varying descriptor system stability is mainly Lyapunov stability; however, Lyapunov stability deals with the whole state performance of the system, but it does not reflect a transient performance of the system. The transient performance refers to the system stability in a short period, but it is different from Lyapunov stability. In engineering, a whole stability of the system may have a very bad transient performance, which would cause a bad effect in the engineering. Therefore, people tend to be more concerned about the transient performance of the system [1-5] than the overall steady state performance of the system.

In order to study the transient performance of the system, scholars have given the concept of finite-time stability. Finitetime stability of time-varying descriptor system is a new field; scholars have mainly studied the finite-time stability of linear system for years and also made some corresponding results. For example, [6-10] proposed the definition of finite-time stability and finite-time boundedness; [5-8] proposed the sufficient conditions for the finite-time stability of general linear system; $[1,11]$ studied the input and output finite-time stability for time-varying descriptor system.

In addition, a finite-time stability problem with external disturbance is called finite-time boundedness; the concept of finite-time boundedness came from finite-time stability. We have some preliminary research results about the finitetime boundedness. For example, [12-14] studied the finitetime bounded problem of the linear time-varying system with impulse; [15-18] proposed the design methods of dynamic compensators; [19-21] discussed the finite-time control problems of uncertain system with disturbance. Scholars introduced the definition of finite-time stability and have given the necessary and sufficient conditions for finite-time stability of the descriptor system, but the finite-time stability problems 
of time-varying descriptor system have no available research results, especially when $E(t)$ is time-variant. So, it is necessary to study finite-time stability of the time-varying uncertain descriptor system.

The paper is divided into two parts: firstly, we deals with the problem of finite-time stability of time-varying descriptor system. We give the necessary and sufficient condition of finite-time stability and finite-time boundedness of system with time-varying function matrix $E(t)$; the unstable system can be controlled by the state feedback controllers. We also make the corresponding research for time-varying uncertain descriptor system. Secondly, we design a finite-time boundedness state feedback controller for time-varying uncertain descriptor system to make the close-loop system finite-time boundedness.

The paper is organized as follows. In Section 2, we give some results of finite-time stability and boundedness for time-varying descriptor system. In Section 3, some results of time-varying uncertain descriptor system are provided. In Section 4, a numerical example is presented to illustrate the efficiency of the proposed result.

\section{Finite-Time Control of Time-Varying Descriptor Systems}

\subsection{Finite-Time Stable of Time-Varying Descriptor Systems}

Definition 1. Time-varying matrix $E(t)$ is singular on time interval $[0, T]$, if there exists a $t \in[0, T]$ such that $\operatorname{rank} E(t)<$ $n$.

Consider the following time-varying descriptor systems

$$
E(t) \dot{x}(t)=A(t) x(t),
$$

where $x(t) \in R^{n}$ is the state. $A(\cdot)$ is given continuous matrixvalued function. $E(t) \in R^{n}$ is singular on time interval $[0, T]$.

Definition 2. The time-varying descriptor system (1) is said to be finite-time stable with respect to $\left(c_{1}, c_{2}, T, R(t)\right)$ with positive definite matrix $R(t)$, and given three positive scalars $c_{1}, c_{2}, T$, with $c_{1}<c_{2}$, if $x^{T}(0) E^{T}(0) R(0) E(0) x(0) \leq c_{1}$, then $x^{T}(t) E^{T}(t) R(t) E(t) x(t)<c_{2}$, for all $t \in[0, T]$.

Theorem 3. The following statements are equivalent:

(i) system (1) is FTS respect to $\left(c_{1}, c_{2}, T, R(t)\right)$.

(ii) For all $t \in[0, T], \Phi^{T}(t, 0) E^{T}(t) R(t) E(t)<$ $\left(c_{2} / c_{1}\right) E^{T}(0) R(0) E(0)$, where $\Phi(t, 0)$ is the state transition matrix and $R(t)$ is positive definite matrix.

(iii) For all $t \in[0, T]$, the differential Lyapunov inequality, with terminal and initial conditions

(a) $M(t)<0$, where

$$
\begin{aligned}
M(t)= & A^{T}(t) P(t) E(t)+E^{T}(t) P(t) A(t) \\
& +\dot{E}^{T}(t) P(t) E(t)+E^{T}(t) \dot{P}(t) E(t) \\
& +E^{T}(t) P(t) \dot{E}(t)
\end{aligned}
$$

(b) $R(t) \leq P(t) \leq P(0)<\left(c_{2} / c_{1}\right) R(0), R(t)$ is positive definite matrix, admits a piecewise continuously differentiable symmetric solution $p(\cdot)$.

Proof. (ii) $\Rightarrow$ (i) Let $x^{T}(0) E^{T}(0) R(0) E(0) x(0) \leq c_{1}$; assume $x(t)=\Phi(t, 0) x(0)$, have

$$
\begin{aligned}
x^{T} & (t) E^{T}(t) R(t) E(t) x(t) \\
& =x^{T}(0) \Phi^{T}(t, 0) E^{T}(t) R(t) E(t) x(0) \\
& <\frac{c_{2}}{c_{1}} E^{T}(0) R(0) E(0) \\
& <c_{2} .
\end{aligned}
$$

Therefore, system (1) is FTS.

(i) $\Rightarrow$ (ii) by contradiction. Let us assume $\exists \bar{t}, \bar{x}$

$$
\begin{gathered}
\bar{x}^{T}(\bar{t}) \Phi^{T}(\bar{t}, 0) E^{T}(\bar{t}) R(\bar{t}) E(\bar{t}) \Phi(\bar{t}, 0) \bar{x}(\bar{t}) \\
\geq \frac{c_{2}}{c_{1}} \bar{x}^{T}(0) E^{T}(0) R(0) E(0) \bar{x}(0) .
\end{gathered}
$$

Let $x^{T}(0) E^{T}(0) R(0) E(0) x(0)=c_{1}, \exists \lambda$, such that $\bar{x}(t)=\lambda x(0)$; then (4) implies that

$$
x^{T}(0) \Phi^{T}(\bar{t}, 0) E^{T}(\bar{t}) R(\bar{t}) E(\bar{t}) \Phi(\bar{t}, 0) x(0) \geq c_{2} ;
$$

therefore

$$
\begin{aligned}
x^{T} & (\bar{t}) E^{T}(\bar{t}) R(\bar{t}) E(\bar{t}) x(\bar{t}) \\
\quad & x^{T}(0) \Phi^{T}(\bar{t}, 0) E^{T}(\bar{t}) R(\bar{t}) E(\bar{t}) \Phi(\bar{t}, 0) x(0) \\
& \geq c_{2} .
\end{aligned}
$$

Obviously, it contradicts the initial assumption that system (1) is FTS.

(iii) $\Rightarrow$ (i) Let $V(t, x)=x^{T}(t) E^{T}(t) P(t) E(t) x(t), \dot{V}(t, x)=$ $x^{T}(t) M(t) x(t)$.

Then (a) implies that $\dot{V}(t, x)$ is negative definite along the trajectories of system (1).

Now $x^{T}(0) E^{T}(0) R(0) E(0) x(0) \leq c_{1}$, then for a generic $t$, such that

$$
\begin{aligned}
& x^{T}(t) E^{T}(t) R(t) E(t) x(t) \\
& \quad \leq x^{T}(t) E^{T}(t) P(t) E(t) x(t) \\
& \quad<x^{T}(0) E^{T}(0) P(0) E(0) x(0) \\
& \quad \leq \frac{c_{2}}{c_{1}} x^{T}(0) E^{T}(0) R(0) E(0) x(0) \\
& \quad \leq c_{2} .
\end{aligned}
$$

(i) $\Rightarrow$ (iii) By contradiction. Because system (1) is FTS. Let $z=\epsilon x$ for a small $\epsilon>0$, for all $t \in[0, T]$, such that

$$
\begin{aligned}
& x^{T}(0) E^{T}(0) P(0) E(0) x(0) \leq c_{1} \\
& \quad \Longrightarrow x^{T}(t) E^{T}(t) R(t) E(t) x(t)+\|z\|_{2}^{2}<c_{2} .
\end{aligned}
$$


Let $P(\cdot)$ be the solution of

$$
\begin{gathered}
M(t)=\epsilon^{2} I, \\
R(t)=P(t) .
\end{gathered}
$$

And assume that $\exists \bar{x}$, such that

$$
\bar{x}^{T}(t) E^{T}(t) P(0) E(t) \bar{x}(t) \geq \frac{c_{2}}{c_{1}} \bar{x}^{T}(t) E^{T}(t) R(0) E(t) \bar{x}(t) .
$$

Now let $x^{T}(0) E^{T}(0) R(0) E(0) x(0)=c_{1}, \exists \lambda$, such that $\bar{x}(t)=$ $\lambda x(0)$.

Then (11) implies

$$
x^{T}(0) E^{T}(0) R(0) E(0) x(0) \geq c_{2} ;
$$

from (9) we obtain that

$$
\frac{d}{d t} x^{T}(t) E^{T}(t) P(t) E(t) x(t)=-\epsilon^{2} x^{T}(t) x(t) .
$$

Integrating (13) from 0 to $t$ we have

$$
\begin{aligned}
& x^{T}(t) E^{T}(t) P(t) E(t) x(t)-x^{T}(0) E^{T}(0) R(0) E(0) x(0) \\
& \quad=-\epsilon^{2}\|x\|_{2}^{2} .
\end{aligned}
$$

Therefore

$$
\begin{aligned}
& x^{T}(t) E^{T}(t) R(t) E(t) x(t) \\
& \quad \geq x^{T}(t) E^{T}(t) P(t) E(t) x(t) \\
& \quad=x^{T}(0) E^{T}(0) P(0) E(0) x(0)-\epsilon^{2}\|x\|_{2}^{2} \\
& \quad \geq c_{2}-\|z\|_{2}^{2},
\end{aligned}
$$

which contradicts (8).

2.2. Finite-Time Boundedness of Time-Varying Descriptor Systems. Consider the following time-varying descriptor systems

$$
E(t) \dot{x}(t)=A(t) x(t)+G(t) \omega(t)
$$

where $x(t) \in R^{n}$ is the state and $\omega(t) \in R^{l}$ is exogenous input. $A(t) \in R^{n \times n}, G(t) \in R^{n \times l}$ are given constant matrices. $E(t) \in$ $R^{n \times n}$ is a singular function matrix, and rank $E(t)=q<n$.

The exogenous disturbance $\omega(t)$ is time varying and satisfies the constraint

$$
\int_{0}^{T} \omega^{T}(t) \omega(t) d t \leq d, \quad d \geq 0 .
$$

Definition 4. The system (16) subject to an exogenous disturbance $\omega(t)$ satisfies (17) and is said to be finite-time bounded with respect to $\left(c_{1}, c_{2}, T, R(t), d\right)$ with positive definite matrices $R(t)$, and given three positive scalars $c_{1}, c_{2}, T$, with $c_{1}<c_{2}$, if $x^{T}(0) E^{T}(0) R(0) E(0) x(0) \leq c_{1}$, then $x^{T}(t) E^{T}(t) R(t) E(t) x(t)<c_{2}$.
Theorem 5. The time-varying descriptor system (16) is finitetime bounded, if there exist two positive definite nonsingular matrices $P(t) \in R^{n \times n}, Q \in R^{l \times l}$, such that

$$
\begin{gathered}
{\left[\begin{array}{cc}
M(t) & E^{T}(t) P(t) G(t) \\
G^{T}(t) P^{T}(t) E(t) & Q
\end{array}\right]<0,} \\
R(t) \leq P(t) \leq P(0) \leq R(0), \\
c_{1}+d \lambda_{\max }(Q)<c_{2}
\end{gathered}
$$

hold, where $\lambda_{\max }(\cdot)$ denote the maximum eigenvalue of the argument,

$$
\begin{aligned}
M(t)= & A^{T}(t) P(t) E(t)+E^{T}(t) P(t) A(t) \\
& +\dot{E}^{T}(t) P(t) E(t)+E^{T}(t) \dot{P}(t) E(t) \\
& +E^{T}(t) P(t) \dot{E}(t) .
\end{aligned}
$$

Proof. Let $V(t, x)=x^{T}(t) E^{T}(t) P(t) E(t) x(t)$.

We have

$$
\begin{aligned}
\dot{V}(t, x)= & x^{T}(t) M(t) x(t)+\omega^{T}(t) G^{T}(t) P^{T}(t) E(t) x(t) \\
& +x^{T}(t) E^{T}(t) P(t) G(t) \omega(t) \\
= & \left(x^{T}(t), \omega^{T}(t)\right) \\
& \times\left[\begin{array}{cc}
M(t) & E^{T}(t) P(t) G(t) \\
G^{T}(t) P^{T}(t) E(t) & Q
\end{array}\right] \\
& \times\left(\begin{array}{c}
x(t) \\
\omega(t)
\end{array}\right)-\omega^{T}(t) Q \omega(t) \\
< & 0 .
\end{aligned}
$$

Therefore,

$$
\dot{V}(t, x)<\omega^{T}(t) Q \omega(t)
$$

integrating from 0 to $t$, with $t \in[0, T]$, we have

$$
V(t, x)<V_{0}+d \lambda_{\max }(Q)
$$

From (19), we have

$$
\begin{aligned}
x^{T} & (t) E^{T}(t) R(t) E(t) x(t) \\
& \leq V(t, x)<V(0, x)+d \lambda_{\max }(Q) \\
& <x^{T}(0) E^{T}(0) R(0) E(0) x(0)+d \lambda_{\max }(Q) \\
& <c_{1}+d \lambda_{\max }(Q) \\
& <c_{2} .
\end{aligned}
$$

Therefore, system (16) is FTB. The proof is completed. 
2.3. Finite-Time Bounded Control of Time-Varying Descriptor Systems. Consider the following time-varying descriptor systems

$$
E(t) \dot{x}(t)=A(t) x(t)+B(t) u(t)+G(t) \omega(t),
$$

where $x(t) \in R^{n}$ is the state; $u(t) \in R^{m}$ is control input; $\omega(t) \epsilon$ $R^{l}$ is exogenous input. $\left.A(t) \in R^{n \times n}, B(t)\right) \in R^{n \times m} G(t) \in R^{n \times l}$ are given continuous matrix-valued functions. $E(t) \in R^{n \times n}$ is a singular function matrix, and rank $E(t)<n$.

Find a state feedback control law

$$
u(t)=K(t) x(t),
$$

where $K(t) \in R^{m \times n}$. Let $A_{c}(t)=(A(t)+B(t) K(t))$. Then the closed-loop system is given by

$$
E(t) \dot{x}(t)=A_{c}(t) x(t)+G(t) \omega(t) .
$$

Theorem 6. The time-varying descriptor system (19) is finitetime bounded, if there exist a symmetric positive definite matrix $P(t) \in R^{n \times n}$, and a nonsingular matrix $Q \in R^{l \times l}$, such that inequality (19), (20) and the following condition hold:

$$
\left[\begin{array}{cc}
M(t) & E^{T}(t) P(t) G(t) \\
G^{T}(t) P^{T}(t) E(t) & -Q^{T}
\end{array}\right]<0 .
$$

Moreover, the state feedback control law is given by

$$
K(t)=B^{-1}(t) G(t) Q^{-1}(t) G^{T}(t) P^{T}(t) E(t),
$$

where $\lambda_{\max }(\cdot)$ denote the maximum eigenvalue of the argument,

$$
\begin{aligned}
M(t)= & A^{T}(t) P(t) E(t)+E^{T}(t) P(t) A(t) \\
& +\dot{E}^{T}(t) P(t) E(t)+E^{T}(t) \dot{P}(t) E(t) \\
& +E^{T}(t) P(t) \dot{E}(t) .
\end{aligned}
$$

Proof. From Theorem 5, the conditions for FTB is that there exist a nonsingular matrix $Q$ such that (19), (20) and the following matrix inequality hold:

$$
\left[\begin{array}{cc}
\bar{M}(t) & E^{T}(t) P(t) G(t) \\
G^{T}(t) P^{T}(t) E(t) & Q
\end{array}\right]<0,
$$

where

$$
\begin{aligned}
\bar{M}(t)= & (A(t)+B(t) K(t))^{T} P(t) E(t) \\
& +E^{T}(t) P(t)(A(t)+B(t) K(t)) \\
& +\dot{E}^{T}(t) P(t) E(t)+E^{T}(t) \dot{P}(t) E(t) \\
& +E^{T}(t) P(t) \dot{E}(t) .
\end{aligned}
$$

According to Schur's theorem, it is easy to see that (32) can be rewritten as

$$
\bar{M}(t)-E^{T}(t) P(t) G(t) Q^{-1}(t) G^{T}(t) P^{T}(t) E(t)<0 .
$$

Using (30), (34), we have

$$
M(t)+E^{T}(t) P(t) G(t) Q^{-T}(t) G^{T}(t) P(t) E(t)<0 .
$$

Since $P(t)$ is symmetric, then

$$
M(t)+E^{T}(t) P(t) G(t) Q^{-T}(t) G^{T}(t) P^{T}(t) E(t)<0 .
$$

By Schur's theorem, (36) is equivalent to (29). The proof is completed.

2.4. Finite-Time Bounded Control of Time-Varying Uncertain Descriptor Systems. Consider the following time-varying descriptor systems

$$
\begin{aligned}
E(t) \dot{x}(t)= & (A(t)+\Delta A(t) x(t)) \\
& +(B(t)+\Delta B(t)) u(t)+G(t) \omega(t),
\end{aligned}
$$

where $x(t) \in R^{n}$ is the state; $u(t) \in R^{m}$ is control input; $\omega(t) \in$ $R^{l}$ is exogenous input. $A(t) \in R^{n \times n}, B(t) \in R^{n \times m}, G(t) \in R^{n \times l}$ are given continuous matrix-valued functions. $E(t) \in R^{n \times n}$ is a singular function matrix, and rank $E(t)<n$.

$$
[\Delta A(t) \quad \Delta B(t)]=H F\left[E_{1}(t) E_{2}(t)\right]
$$

where $F \in R^{q \times s}$ is unknown and satisfies

$$
F^{T} F \leq I
$$

Theorem 7. The time-varying descriptor system (37) is finitetime bounded, if there exist a symmetric positive definite matrix $P(t) \in R^{n \times n}$, and a nonsingular matrix $Q \in R^{l \times l}$, such that inequality (19), (20), and (32) hold, and the state feedback control law is given by

$$
\begin{aligned}
K(t)= & \left(B(t)+H F E_{2}(t)\right)^{-1} \\
& \times\left(G(t) Q^{-1}(t) G^{T}(t) P^{T}(t) E(t)-H F E_{1}(t)\right),
\end{aligned}
$$

where

$$
\begin{aligned}
\bar{M}(t)= & {[A(t)+\Delta A(t)+(B(t)+\Delta B(t)) K(t)]^{T} } \\
& \times P(t) E(t)+E^{T}(t) P(t) \\
& \times[A(t)+\Delta A(t)+(B(t)+\Delta B(t)) K(t)] \\
& +\dot{E}^{T}(t) P(t) E(t)+E^{T}(t) \dot{P}(t) E(t) \\
& +E^{T}(t) P(t) \dot{E}(t) .
\end{aligned}
$$

Proof. Applying (40) to (34), and $P(t)$ is symmetric, then the proof is completed. 


\section{Numerical Example}

Consider a time-varying descriptor system (26) with

$$
\begin{array}{cc}
E=\left[\begin{array}{ll}
t & 0 \\
0 & 0
\end{array}\right], \quad A=\left[\begin{array}{cc}
t & 0 \\
0 & -1
\end{array}\right], \quad B=\left[\begin{array}{ll}
1 & 0 \\
0 & t
\end{array}\right], \\
G=\left[\begin{array}{c}
1 \\
-t
\end{array}\right] .
\end{array}
$$

Let $R(t)=P(t), Q=t, T=1$. Exist $P(t)=\left[\begin{array}{cc}-t & 0 \\ 0 & t\end{array}\right]$, such that

$$
\begin{gathered}
M(t)=\left[\begin{array}{cc}
-2 t^{3}-3 t^{2} & 0 \\
0 & 0
\end{array}\right], \quad E^{T}(t) P(t) G(t)=\left[\begin{array}{c}
-t^{2} \\
0
\end{array}\right], \\
G^{T}(t) P^{T}(t) E(t)=\left[\begin{array}{ll}
-t^{2} & 0
\end{array}\right] .
\end{gathered}
$$

So, condition (29), (19), (20) hold. the system (32) is finitetime bounded and the state feedback controller with

$$
K(t)=B^{-1}(t) G(t) Q^{-1}(t) G^{T}(t) P^{T}(t) E(t)=\left[\begin{array}{cc}
-t & 0 \\
1 & 0
\end{array}\right] .
$$

\section{Conclusions}

In this paper, we have studied the finite-time stability and given a sufficient and necessary condition of the finite-time stability; the state feedback controller was designed, then we studied the finite-time boundedness of time-varying descriptor systems, and a sufficient and necessary condition of the finite-time boundedness is given and a state feedback controller was designed. In the end, a numerical example is given to prove the effectiveness of the method.

\section{Acknowledgment}

This project was supported by the National Nature Science Foundation of China (no. 61074005), the Talent Project of the High Education of Liaoning province, China, under Grant no. LR2012005.

\section{References}

[1] J. Yao, Z.-H. Guan, G. Chen, and D. W. C. Ho, "Stability, robust stabilization and $H_{\infty}$ control of singular-impulsive systems via switching control," Systems \& Control Letters, vol. 55, no. 11, pp. 879-886, 2006.

[2] X. Lin, H. Du, and S. Li, "Finite-time boundedness and $L_{2}$ gain analysis for switched delay systems with norm-bounded disturbance," Applied Mathematics and Computation, vol. 217, no. 12, pp. 5982-5993, 2011.

[3] G. Garcia, S. Tarbouriech, and J. Bernussou, "Finite-time stabilization of linear time-varying continuous systems," IEEE Transactions on Automatic Control, vol. 54, no. 2, pp. 364-369, 2009.

[4] Z.-H. Guan, C. W. Chan, A. Y. T. Leung, and G. Chen, "Robust stabilization of singular-impulsive-delayed systems with nonlinear perturbations," IEEE Transactions on Circuits and Systems, vol. 48, no. 8, pp. 1011-1019, 2001.
[5] Y. Tong, B. Wu, and F. Huang, "Finite-time bound and $L L_{2}$-gain analysis for linear time-varying singular impulsive systems," in Proceedings of the Chinese Control and Decision Conference (CCDC '12), no. 24, 2012.

[6] E. Moulay, M. Dambrine, N. Yeganefar, and W. Perruquetti, "Finite-time stability and stabilization of time-delay systems," Systems \& Control Letters, vol. 57, no. 7, pp. 561-566, 2008.

[7] J. Yao, J.-e. Feng, L. Sun, and Y. Zheng, "Input-output finitetime stability of time-varying linear singular systems," Journal of Control Theory and Applications, vol. 10, no. 3, pp. 287-291, 2012.

[8] F. Amato, R. Ambrosino, C. Cosentino, and G. DeTommasi, "Input-output finite-time stability of linear systems," in Proceedings of the 17th Mediterranean Conference on Control and Automation, pp. 342-346, Hessaloniki, Greece, June 2009.

[9] F. Amato, M. Ariola, C. Cosentino, C. T. Abdallah, and P. Dorato, "Necessary and sufficient conditions for finite-time stability of linear systems," in Proceedings of the American Control Conference, pp. 4452-4456, Denver, Colo, USA, June 2003.

[10] N. A. Kablar and D. L. Debeljkovic, "Finite-time stability of time-varying linear singular systems," in Proceedings of the 37th IEEE Conference on Decision and Control (CDC '98), pp. 38313836, Tampa, Fla, USA, December 1998.

[11] J. Xu and J. Sun, "Finite-time stability of linear time-varying singular impulsive systems," IET Control Theory \& Applications, vol. 4, no. 10, pp. 2239-2244, 2010.

[12] S. Zhao, J. Sun, and L. Liu, "Finite-time stability of linear timevarying singular systems with impulsive effects," International Journal of Control, vol. 81, no. 11, pp. 1824-1829, 2008.

[13] F. Amato, M. Ariola, and C. Cosentino, "Finite-time stability of linear time-varying systems: analysis and controller design," IEEE Transactions on Automatic Control, vol. 55, no. 4, pp. 10031008,2010

[14] F. Amato, R. Ambrosino, M. Ariola, and C. Cosentino, "Finitetime stability of linear time-varying systems with jumps," Automatica A, vol. 45, no. 5, pp. 1354-1358, 2009.

[15] F. Amato, M. Ariola, and C. Cosentino, "Finite-time stabilization via dynamic output feedback," Automatica A, vol. 42, no. 2, pp. 337-342, 2006.

[16] F. Amato, M. Ariola, M. Carbone, and C. Cosentino, "Finitetime output feedback control of linear systems via differential linear matrix conditions," in Proceedings of the 45th IEEE Conference on Decision and Control (CDC '06), pp. 5371-5375, San Diego, Calif, USA, December 2006.

[17] P. Dorato, "Short time stability in linear time-varying systems," in Proceedings of the IRE International Convention Record, pp. 83-87, 1961.

[18] J. Sun and Z. Cheng, "Finite-time control for one kind of uncertain linear singular systems subject to norm bounded uncertainties," in Proceedings of the 5th World Congress on Intelligent Control and Automation, pp. 980-984, Hangzhou, Chin, June 2004.

[19] F. Amato, M. Ariola, and P. Dorato, "Finite-time control of linear systems subject to parametric uncertainties and disturbances," Automatica, vol. 37, no. 9, pp. 1459-1463, 2001.

[20] J.-E. Feng, Z. Wu, and J.-B. Sun, "Finite-time control of linear singular systems with parametric uncertainties and disturbances," Acta Automatica Sinica, vol. 31, no. 4, pp. 634-637, 2005.

[21] R. Ambrosino, F. Calabrese, C. Cosentino, and G. de Tommasi, "Sufficient conditions for finite-time stability of impulsive dynamical systems," IEEE Transactions on Automatic Control, vol. 54, no. 4, pp. 861-865, 2009. 


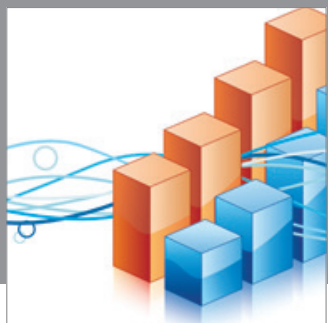

Advances in

Operations Research

mansans

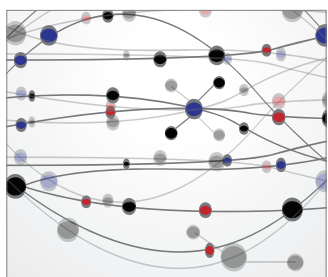

The Scientific World Journal
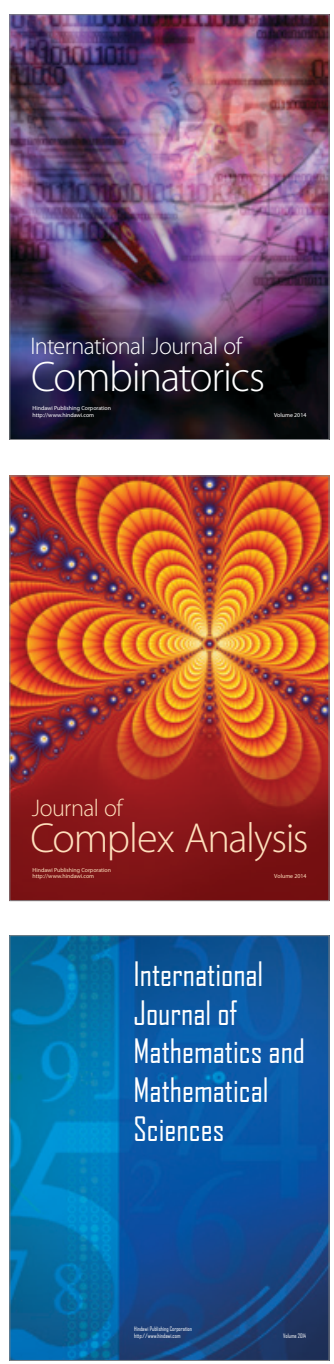
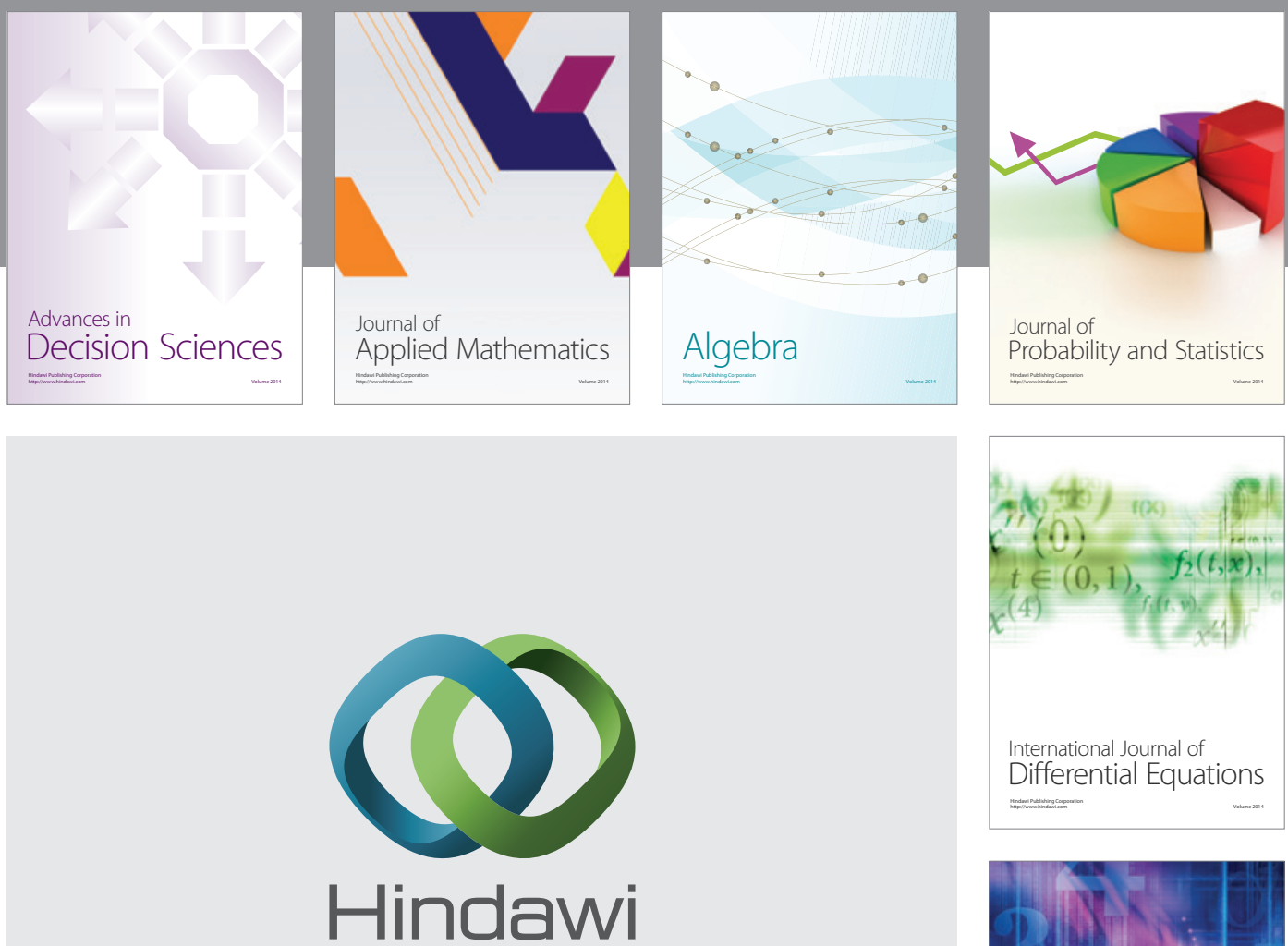

Submit your manuscripts at http://www.hindawi.com
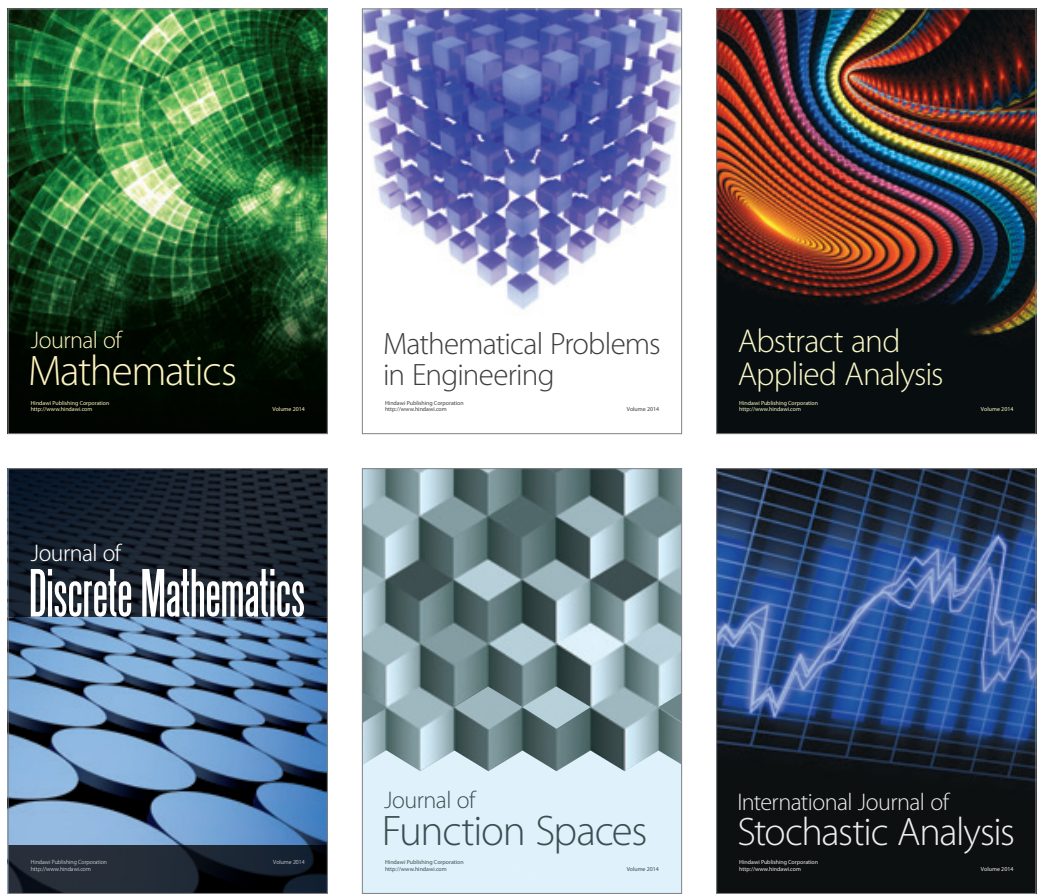

Journal of

Function Spaces

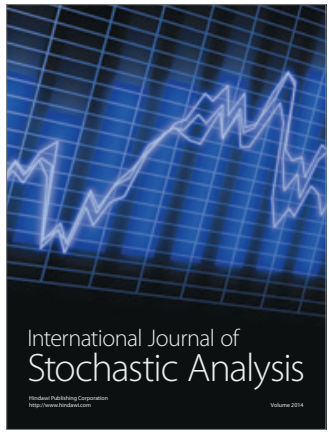

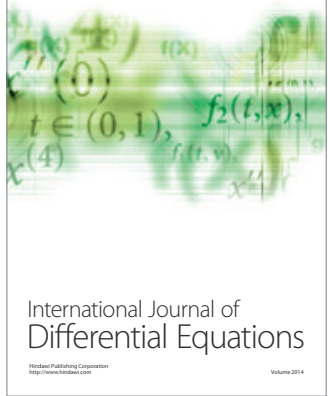
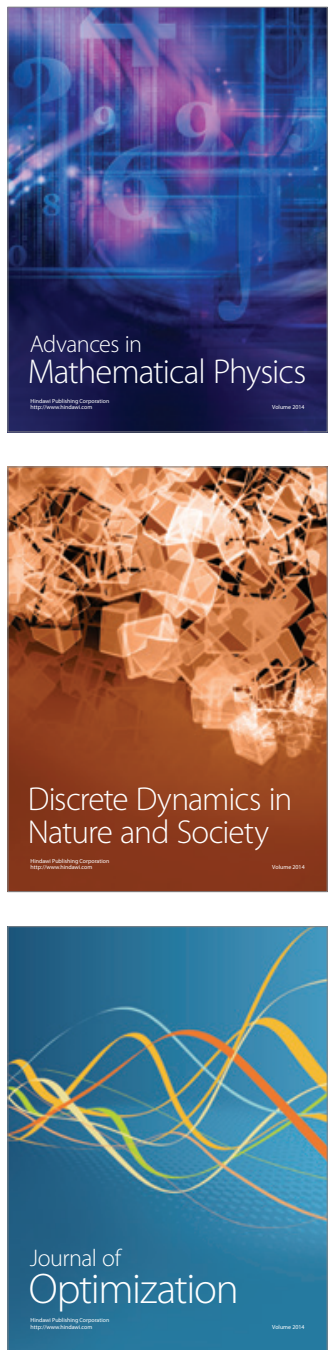